\title{
Spatial Evolution of Resonant Harmonic Mode Triads in a Blasius Boundary Layer
}

\author{
José B. Dávila* \\ Trinity College, Hartford, Connecticut 06106 USA \\ and \\ Rudolph A. King ${ }^{\dagger}$ \\ NASA Langley Research Center, Hampton, Virginia 23681 USA
}

\begin{abstract}
Blasius boundary layer evolution is studied by means of bicoherence calculations. The layer is acoustically excited at the T-S frequency to provide a controlled transition. Measurements are made using a smooth surface as well as various roughness patterns. The bicoherence calculations are used to determine the extent to which frequency resonant velocity fluctuation waves can participate in energy exchange. The emphasis is on downstream variation of the individual interactions among harmonic modes. A limited picture of the role of quadratic wave interactions is revealed.
\end{abstract}

\section{Nomenclature}

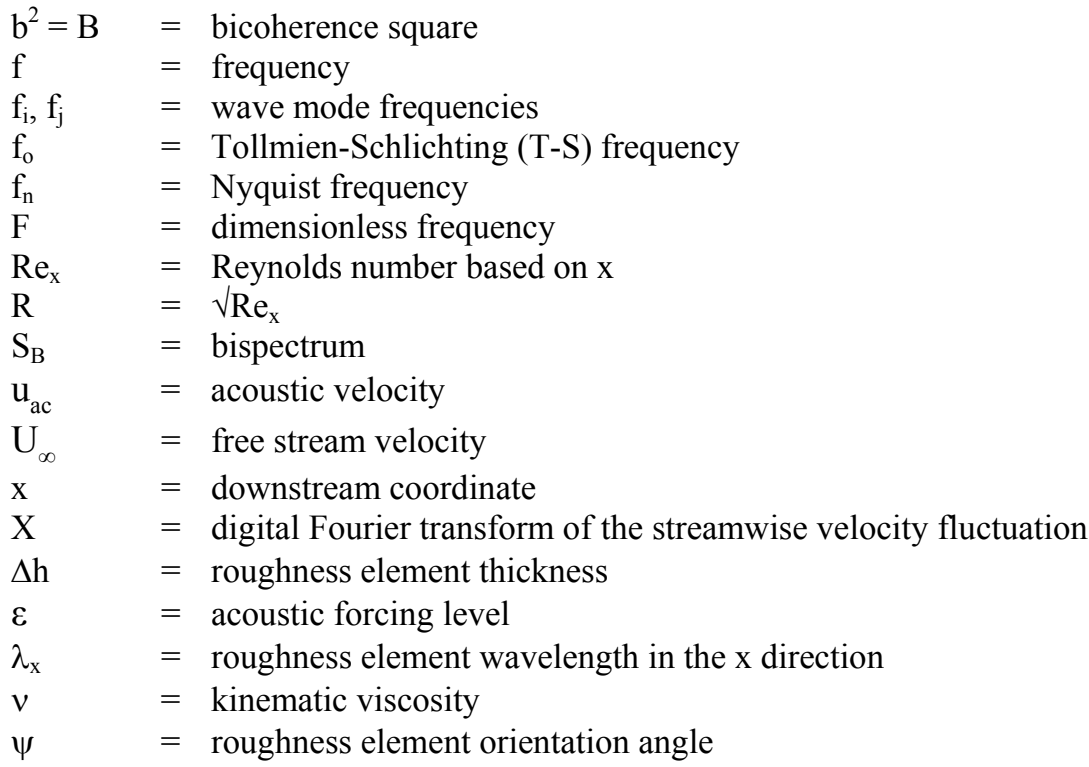

\section{Introduction}

kin friction and heat transfer rates are significantly higher in turbulent flow. The transition to turbulence occurs Supon the onset of non-linear interactions. Advanced time series techniques such as the calculation of the bicoherence are conceived to determine the nature of quadratic interactions. In the early transitioning flow the fundamental Tollmien-Schlichting wave distorts and generates harmonics that interact with one another. King and Breuer $^{1}$ discuss the mechanism of receptivity by which external disturbances determine the wavelike disturbances in

\footnotetext{
${ }^{*}$ Visiting Assistant Professor, Department of Engineering.

${ }^{\dagger}$ Research Engineer, Flow Physics and Control Branch.
} 
the early flow and show amplitude growth data for various acoustic disturbance levels and wall roughness patterns. Results obtained by Dávila, King and Chokani ${ }^{2}$ reveal that the early non-linear interactions persist downstream in narrow frequency ranges. This discreteness makes it difficult to present the data using the often-shown contour graphs. This is especially true for smooth surfaces. In the present work several smooth- and rough-walled cases are studied for acoustically excited transitioning layers by examining the bicoherence value as a function of downstream position for selected interactions. This represents a departure from the more common presentation, away from the panoramic bispectral picture, in favor of individual wave triads.

\section{Experimental Set-Up}

The experiments were conducted in the closed-loop, low-turbulence ( $2 \mathrm{ft}$. by $3 \mathrm{ft}$.) Low-Speed Wind Tunnel located at NASA Langley Research Center at a freestream velocity of $\mathrm{U}_{\infty} \approx 11.1 \mathrm{~m} / \mathrm{s}$. The wind tunnel has a $6.1 \mathrm{~m}$ long test section with a $0.61 \mathrm{~m}$ (high) by $0.91 \mathrm{~m}$ (wide) cross-section. The contraction ratio is $10: 1$ and the measured turbulence intensities are approximately $0.1 \%$ in the range of $0.1<\mathrm{f}<400 \mathrm{~Hz}$. The boundary layer under investigation developed on a $2.62 \mathrm{~m}$ long, $12.7 \mathrm{~mm}$ thick, aluminum plate, with a 24:1 elliptical leading edge, which spanned the width of the test section. The measurement surface was polished to $0.2 \mu \mathrm{m} \mathrm{r.m.s.} \mathrm{surface} \mathrm{finish.} \mathrm{A}$ trailing edge flap was used to position the stagnation point on the measurement surface.

A rectangular cutout, located $24.6 \mathrm{~cm}$ from the leading edge of the plate, is equipped with interchangeable inserts to allow mounting surface "roughness" pattern about Branch I. The patterns consist of a series of thin rectangular strips attached to the insert surface. In all cases, they were of equal width, spaced by their own width, and having a constant thickness $\Delta \mathrm{h}$ (elevation above the plate surface); this produces a square-wave roughness pattern. They were generated using a photolithographic process. Only the medium and high roughness cases, with $\Delta \mathrm{h}=36 \mu \mathrm{m}$, and $\Delta \mathrm{h}=72 \mu \mathrm{m}$, respectively, are shown. Results were also obtained with $\Delta \mathrm{h}=18 \mu \mathrm{m}$, but they showed no special features. The angle $\psi$ is the orientation of the length of the strips with respect to the y axis (the spanwise direction). It is equivalently the misalignment of the surface roughness wavenumber vector with respect to the $\mathrm{x}$ axis (the streamwise direction). In patterns with $\psi=0^{\circ}$ the surface roughness wavenumber points in the direction of flow, thus being two-dimensional. In patterns with $\psi>0^{\circ}$ the wavenumber vector has a component in the spanwise direction; thus a three-dimensionality is introduced. The x-component of the wavenumber has an associated $\mathrm{x}$-direction wavelength $\lambda_{\mathrm{x}}$ that defines the spatial periodicity of the pattern in the streamwise direction. Table 1 summarizes the geometric details of the patterns. The values of $\lambda_{x}$ were chosen to be close to the T-S wavelength, $50.2 \mathrm{~mm}$ at Branch I. Details of the wind tunnel and the roughness patterns are given in reference 1 .

\begin{tabular}{|l|c|c|c|}
\hline pattern & $\lambda_{\mathrm{x}}[\mathrm{mm}]$ & $\psi$ & $\Delta \mathrm{h}[\mu \mathrm{m}]$ \\
\hline $\mathrm{C}$ (2-dimensional) & 50.3 & $0^{\circ}$ & 72 \\
\hline $\mathrm{B}$ (2-dimensional) & 50.3 & $0^{\circ}$ & 36 \\
\hline $\mathrm{M}$ (3-dimensional) & 50.7 & $15^{\circ}$ & 36 \\
\hline $\mathrm{K}$ (3-dimensional) & 52.4 & $30^{\circ}$ & 36 \\
\hline $\mathrm{N}$ (3-dimensional) & 55.5 & $45^{\circ}$ & 36 \\
\hline
\end{tabular}

Table 1. Surface roughness pattern geometry details.

Five $203.3 \mathrm{~mm}$ diameter woofers were used for acoustic excitation; four speakers were mounted on the upstream wall and the other on the downstream wall. The amplitude of the acoustic field, $\mathrm{u}_{\mathrm{ac}}$, could be varied to produce different levels of acoustic forcing, $\varepsilon=u_{a c} / U_{\infty}$. More complete details of the experiment are given by King, ${ }^{3}$ and a select group of acoustic and roughness combinations are presented here. Low acoustic levels produced no observable bicoherence. Only medium and high excitation levels, with $\varepsilon=3.8 \times 10^{-5}$ and $\varepsilon=7.6 \times 10^{-5}$, respectively, are discussed here.

The phase-locked velocity fluctuations were derived from the signal of a single-element hot-wire, operated at an overheat ratio of 1.8 with a constant temperature anemometer. The acquisition of the hot-wire signal was triggered with the TTL of a reference signal and sampled at a frequency of $1 \mathrm{kHz}$. The reference signal was the excitation, or forcing frequency, $\mathrm{f}_{0}$, which was applied to the loudspeakers that were used to generate the downstream traveling acoustic wave. The dimensionless frequency of the T-S wave was $F=55 \times 10^{-6}\left(=2 \pi \mathrm{fv} / \mathrm{U}_{\infty}{ }^{2}\right)$, which translates to a physical forcing frequency of $f_{o}=71 \mathrm{~Hz}$. Results are shown for four streamwise stations, given by modified Reynolds number $\mathrm{R}\left(=\sqrt{ } \mathrm{Re}_{\mathrm{x}}\right)=943,1038,1124$ and 1205 with the smooth and rough plates, two-dimensional and 
three-dimensional. While the data are limited, an attempt is made to discern features of the quadratic nonlinearities that are determined by acoustic excitation level and by roughness element thickness and orientation.

\section{Calculations}

The nonlinear energy cascade that occurs due to the three-wave resonant interactions was quantified using the bicoherence. Kim and Powers ${ }^{4}$ give a detailed description of the calculation technique for the bicoherence. Corke and Mangano ${ }^{5}$, Miksad et al. ${ }^{6}$ among others have used the bicoherence to study transition to turbulence, but a few salient details are repeated here for the sake of completeness. The bispectrum is a third-order cumulative function that is defined as:

$$
S_{B}\left(f_{i}, f_{j}\right)=E\left[X *\left(f_{i}\right) X *\left(f_{j}\right) X\left(f_{i}+f_{j}\right)\right]
$$

where $\mathrm{E}[\cdot]$ is the expected value, $\mathrm{X}(\mathrm{f})$ are the digital Fourier transforms of the time series records, the superscript asterisk denotes the complex conjugate. The normalized representation of the bispectrum is the bicoherence, which is bound by 0 and 1 . The bicoherence square is defined by:

$$
b^{2}\left(f_{i}, f_{j}\right)=\frac{\left|S_{B}\left(f_{i}, f_{j}\right)\right|^{2}}{E\left[\left|X\left(f_{i}\right) X\left(f_{j}\right)\right|^{2}\right] E\left[\left|X\left(f_{i}+f_{j}\right)\right|^{2}\right]}
$$

If the triad that is present at $f_{i}, f_{j}$ and the sum mode $f_{i}+f_{j}$ is comprised of independently generated oscillations, then these are statistically independent of each other, and consequently $b^{2}\left(f_{i}, f_{j}\right) \approx 0.0$. On the other hand, high values of the bicoherence indicate a high degree of temporal phase coherence between the product of the oscillations $\left(\right.$ at $f_{i}$ and $f_{j}$ ) and the oscillation at the sum frequency $\left(a t f_{i}+f_{j}\right.$ ). The sum interactions are of the form

$$
\left(\mathrm{f}_{\mathrm{i}}, \mathrm{f}_{\mathrm{j}}\right) \rightarrow \mathrm{f}_{\mathrm{i}}+\mathrm{f}_{\mathrm{j}}
$$

The symbol $\rightarrow$ denotes "phase-coupled interaction with the mode at." The targeted mode is determined by adding the ordinate frequency to the abscissa frequency in a triangular region defined by the vertices $(0,0),\left(f_{n}, 0\right)$, $\left(\mathrm{f}_{\mathrm{n}} / 2, \mathrm{f}_{\mathrm{n}} / 2\right)$, where $\mathrm{f}_{\mathrm{n}}$ is the Nyquist frequency, $500 \mathrm{~Hz}$. This is the top triangular zone of the plot. The difference interactions are of the form,

$$
\left(\mathrm{f}_{\mathrm{i}},-\mathrm{f}_{\mathrm{j}}\right) \rightarrow \mathrm{f}_{\mathrm{i}}-\mathrm{f}_{\mathrm{j}}
$$

which are the ordinate frequency subtracted from the abscissa frequency, are in the region defined by the vertices $(0,0),\left(f_{n}, 0\right),\left(f_{n},-f_{n}\right)$. This is the bottom triangular zone of the plot. The bicoherence square, $b^{2}\left(f_{i}, f_{j}\right)$, with sum and difference interactions is often plotted as a contour level in the $\left(f_{i}, f_{j}\right)$ plane. Figure 1 (reproduced from reference 2 ) is representative of such plots; it is shown here for illustration.

Figure 1 is not typical. In the present results, most contour plots showed narrow peaks involving mostly the fundamental and the harmonics. These are not easily observable; therefore, on this occasion only ten triads, all involving harmonic peaks, were selected for observation. The interactions are defined using the relative frequency $\mathrm{f} / \mathrm{f}_{\mathrm{o}}$, where $\mathrm{f}$ is the frequency and $\mathrm{f}_{\mathrm{o}}$ is the fundamental frequency of $\mathrm{f}_{\mathrm{o}}=71 \mathrm{~Hz}$. The fundamental $71 \mathrm{~Hz}$ and the harmonics $142 \mathrm{~Hz}, 213 \mathrm{~Hz}$ and $284 \mathrm{~Hz}$ correspond to $\mathrm{f} / \mathrm{f}_{\mathrm{o}}$ values of $1,2,3$ and 4 . For convenience, in the discussion of results and in all figures, the bicoherence square is denoted by $B$, in place of $b^{2}$. For example, $B(1,2)=b^{2}\left(f_{o}, 2 f_{o}\right)$, corresponding to a sum interaction targeting the frequency $3 f_{0}$. Similarly, $B(3,-1)=b^{2}\left(3 f_{0},-f_{0}\right)$, corresponding to a difference interaction targeting the frequency $2 \mathrm{f}_{\mathrm{o}}$.

The number of downstream positions where measurements were made precludes proper observation of the expected widening of the bicoherence contours as turbulence breakdown sets in. But in fact, turbulence breakdown does not occur in most of these cases. Instead, the present discussion will focus the downstream variation of the bicoherence values for the selected triads and the corresponding evolution of the energy spectrum.

The boundary layer is excited using an unstable mode of $71 \mathrm{~Hz}$. This forced fundamental mode is clearly identifiable at all streamwise locations; the $60-\mathrm{Hz}$ signal (and its harmonics), due to signals picked up from the electric cables, can also be seen as a series of narrow spikes. 

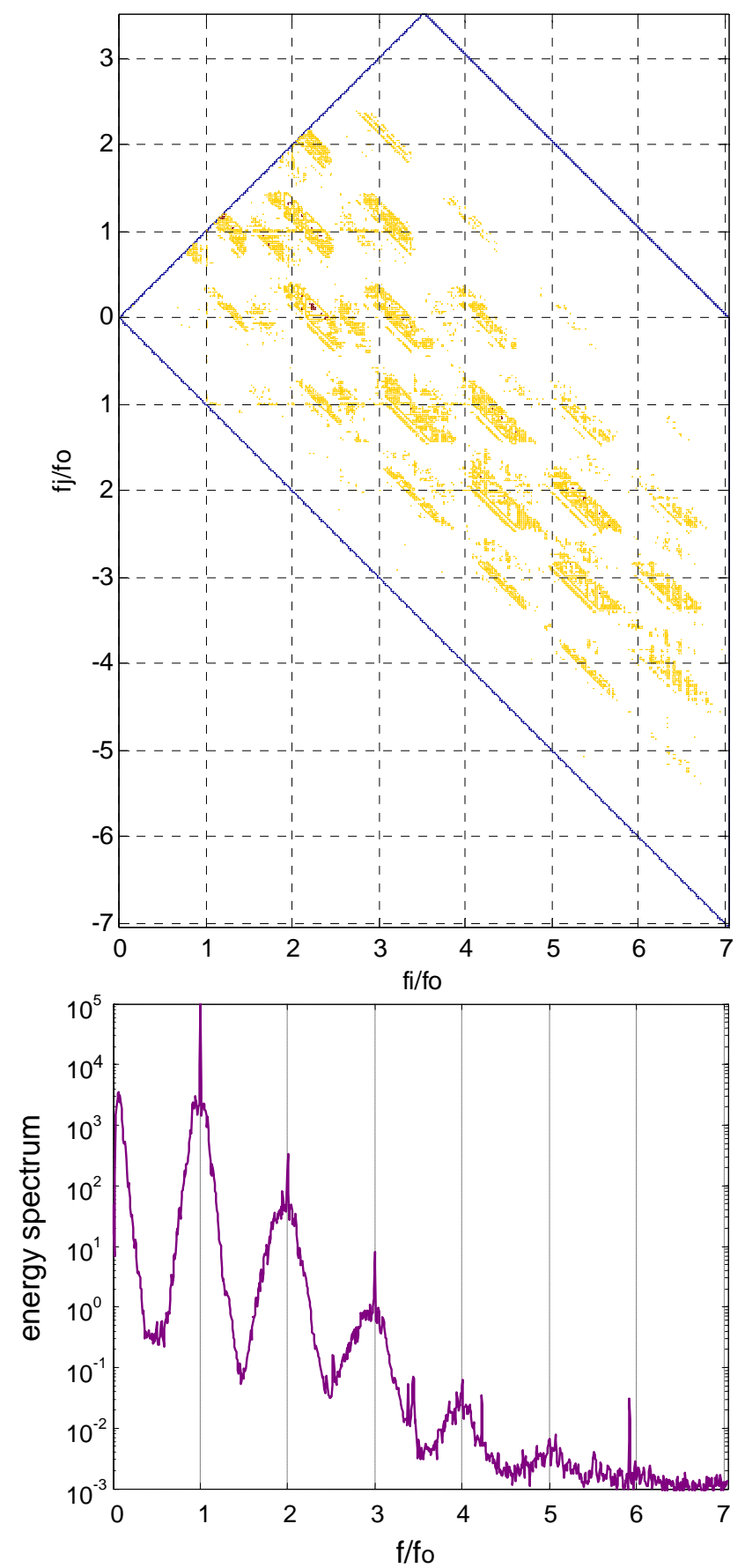

Figure 1. Bicoherence square (top) and Energy Spectrum at $\mathrm{R}=1082$ for the case of high acoustic exitation and high roughness, pattern $\mathrm{C}$. 


\section{Case: Medium excitation, smooth plate}
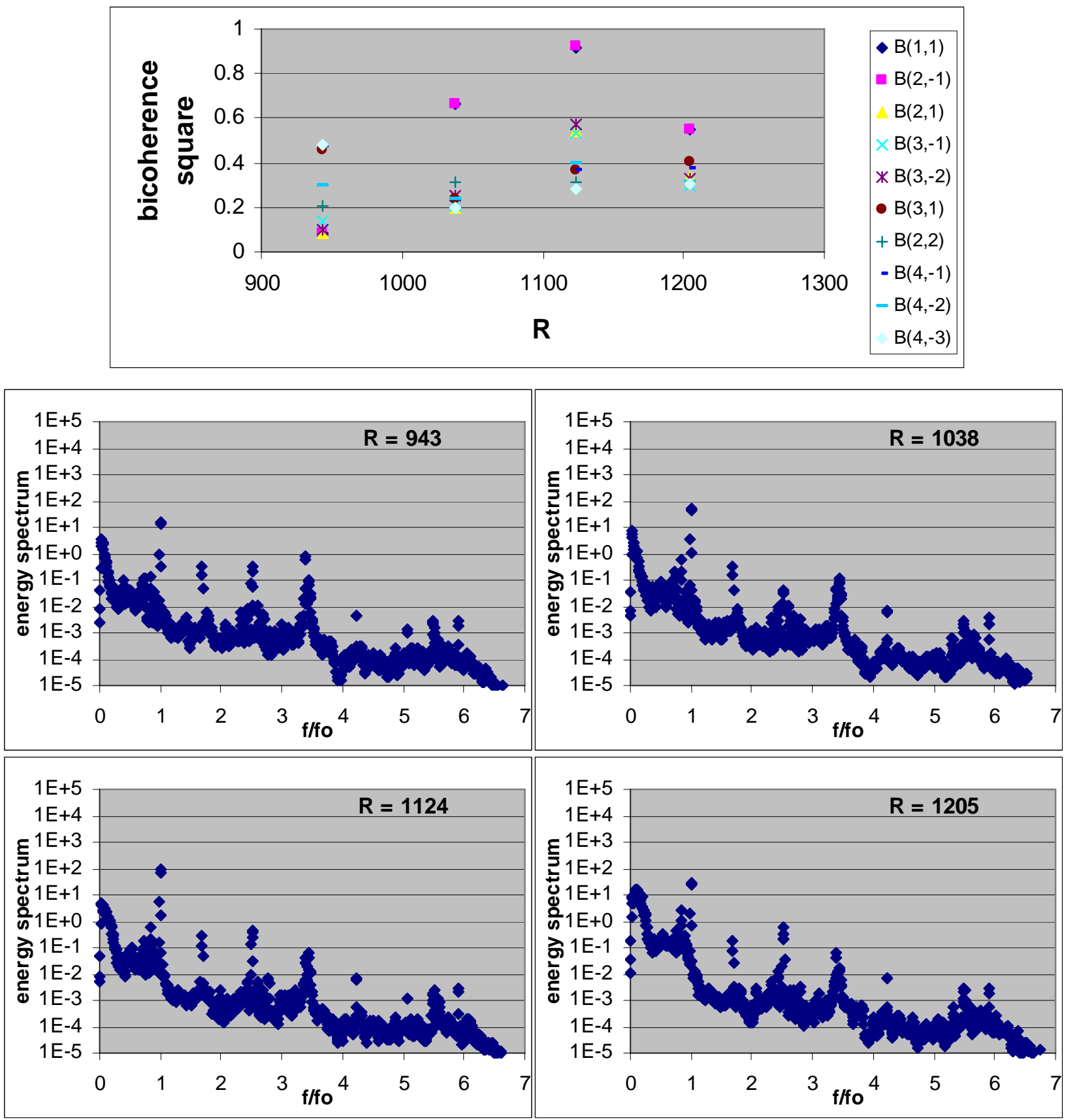

Figure 2. Bicoherence and energy spectrum as a function of $\mathbf{R}$ for the medium-excitation, smooth-plate case.

\section{Results}

Results for for the case of medium acoustic excitation and smooth plate case are shown in figure 2. The energy spectrum is uneventful, and the fundamental and harmonic mode amplitudes are low. The low amplitude of the fundamental prevents it from transferring sufficient energy to the harmonic modes. All triads show increasing bicoherence values up to $R=1124$, but the modes at $f / f_{o}=2,3$, and 4 show low energy values at all stations, indicating poor energy transfer. As a result, at $\mathrm{R}=1205$ the harmonic modes randomize, resulting in lower bicoherence values for all triads. With low bicoherence values at $\mathrm{R}=1205$, energy transfer is inhibited, and therefore the transition does not occur in the $\mathrm{R}$ region studied. 


\section{Case: High excitation, smooth plate}
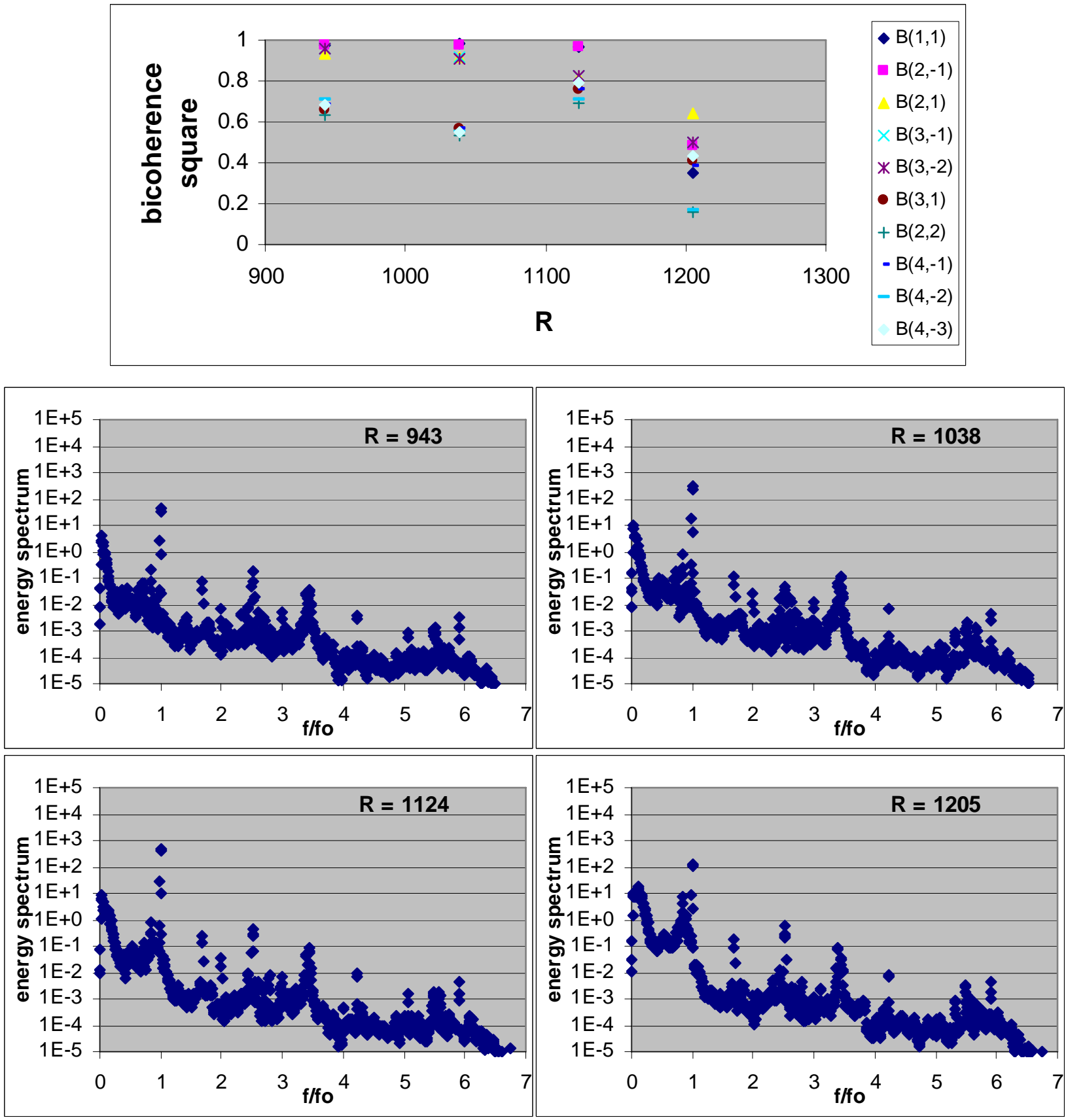

Figure 3. Bicoherence and energy spectrum as a function of $\mathbf{R}$ for the high-excitation, smooth-plate case.

Figure 3 shows the results for the case of high acoustic excitation with a smooth plate. As in the previous case, an uneventful energy spectrum shows that, without surface roughness, the amplitude of the fundamental mode remains low at all stations, despite the high excitation. The triads $(1,1)$ and $(2,-1)$ show high bicoherence due to the growth of the mode at $\mathrm{f} / \mathrm{f}_{\mathrm{o}}=2$. All other triads reach bicoherence values near 0.9 . However, energy transfer to the harmonic modes is not effective, as evidenced by their persistently low harmonic energy levels. With low amplitude levels in the harmonic modes, the bicoherence has decreased for all triads at $\mathrm{R}=1205$. The transition does not occur in the $\mathrm{R}$ region studied. In fact, in both smooth-plate cases, the acoustic excitation alone is not enough to induce transition in the $\mathrm{R}$ region studied. 
Case: Medium excitation, high roughness, $\psi=0^{\circ}$ (pattern C)
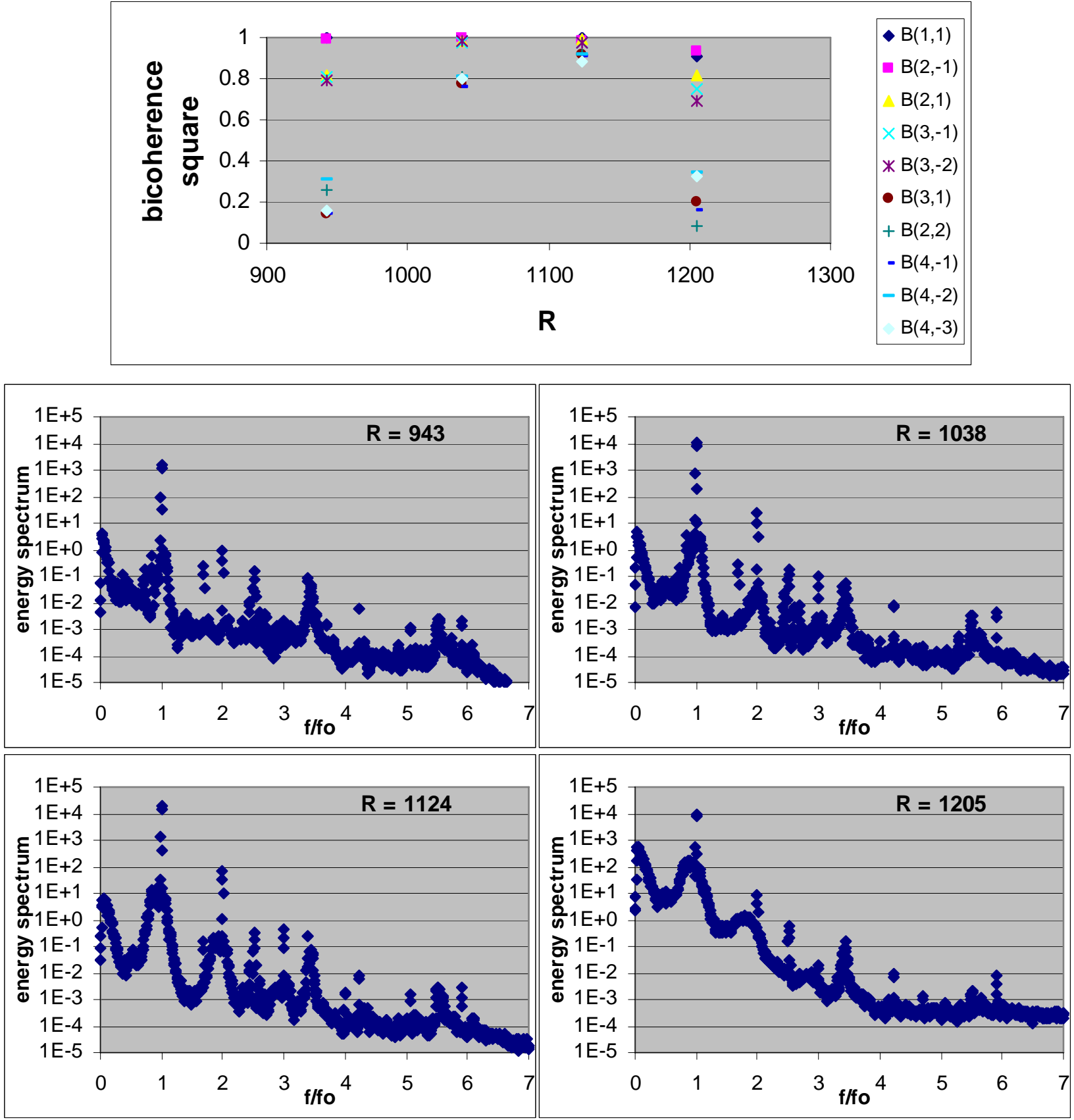

Figure 4. Bicoherence and energy spectrum as a function of $\mathbf{R}$ for the medium-excitation, high-roughness, $\psi=0^{\circ}$ (pattern C).

The results for the medium excitation, high roughness case are shown in figure 4. Evidently the introduction of surface roughness in the receptivity region has resulted in an increase of the fundamental mode amplitude downstream. This behavior is consistent with measurements by King and Breuer ${ }^{1}$ for two-dimensional roughness. The harmonic mode amplitudes are also greater. At $\mathrm{R}=1124$ all triads have high bicoherence, and the harmonic modes (up to $\mathrm{f} / \mathrm{f}_{\mathrm{o}}=4$ ) have grown more than they did in either smooth-plate case, which is evidence of effective energy transfer. At $R=1205$, the filling of the energy valleys shows that transition is incipient. As a result, the higher-frequency harmonic modes begin to randomize, and the bicoherence values begin to decrease. At the last station, for all triads that include the mode at $\mathrm{f} / \mathrm{f}_{\mathrm{o}}=4$, the bicoherence value falls below 0.4 . 


\section{Case: High excitation, high roughness, $\psi=0^{\circ}$ (pattern C)}
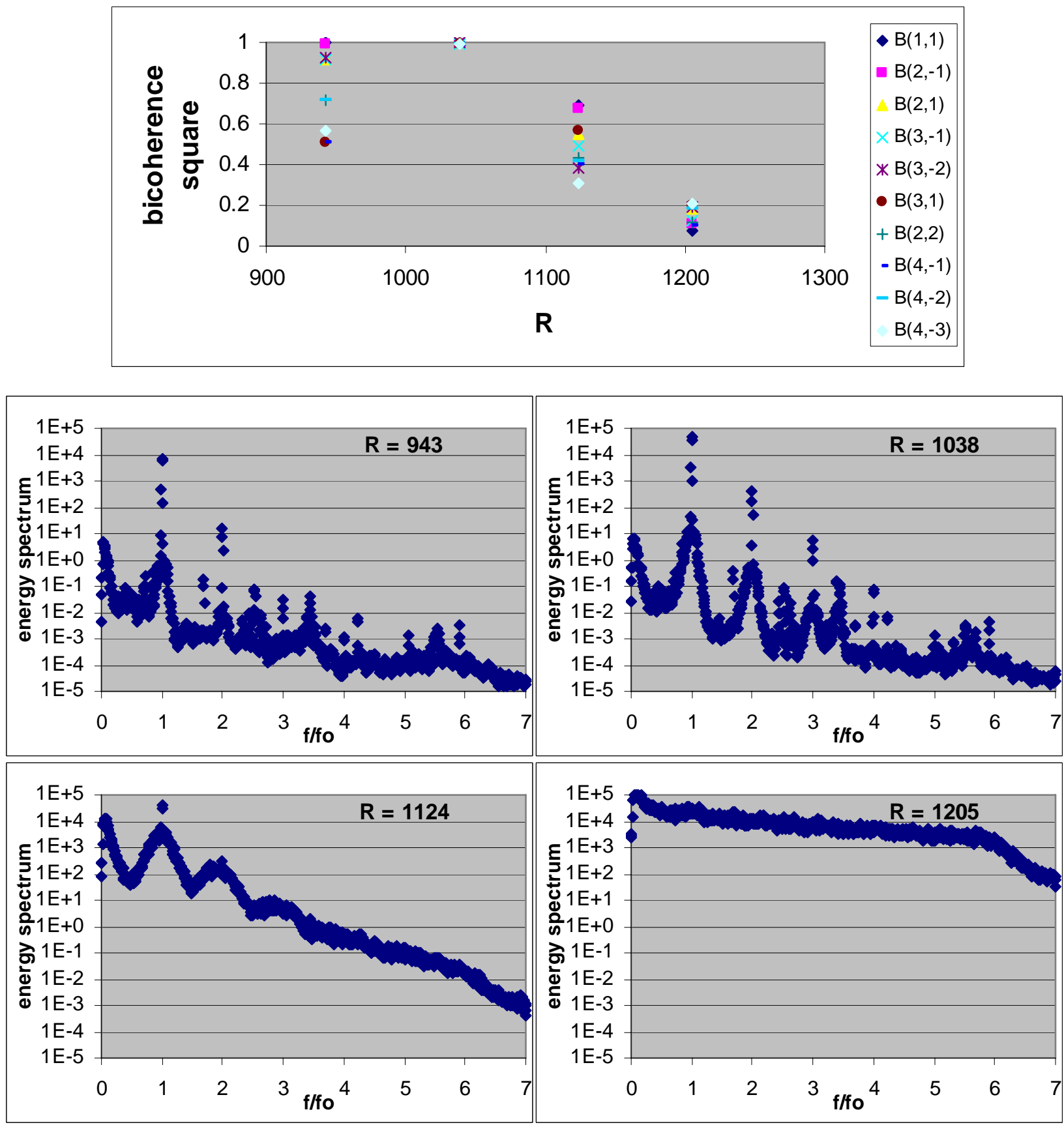

Figure 5. Bicoherence and energy spectrum as a function of $\mathbf{R}$ for the high-excitation, high-roughness, $\psi=\mathbf{0}^{\circ}$ case (pattern C).

The results for the high-excitation, high-roughness case are shown in figure 5. At all stations the fundamental and harmonic modes have higher amplitude than in the high-excitation, smooth case. In addition, all triads reach high values at $\mathrm{R}=1038$, indicating early strong interactions with effective energy transfer to harmonic modes up to $\mathrm{f} / \mathrm{f}_{\mathrm{o}}=4$. Transition has begun at $\mathrm{R}=1124$ and the layer is turbulent at $\mathrm{R}=1205$. The effect of the roughness region is evident: increased mode growth, stronger interactions, effective energy transfer, and early transition.

Data for many patterns were collected at medium acoustic excitation levels. These are shown in the remaining results. 


\section{Case: Medium excitation, medium roughness, $\psi=0^{\circ}$ (pattern B)}
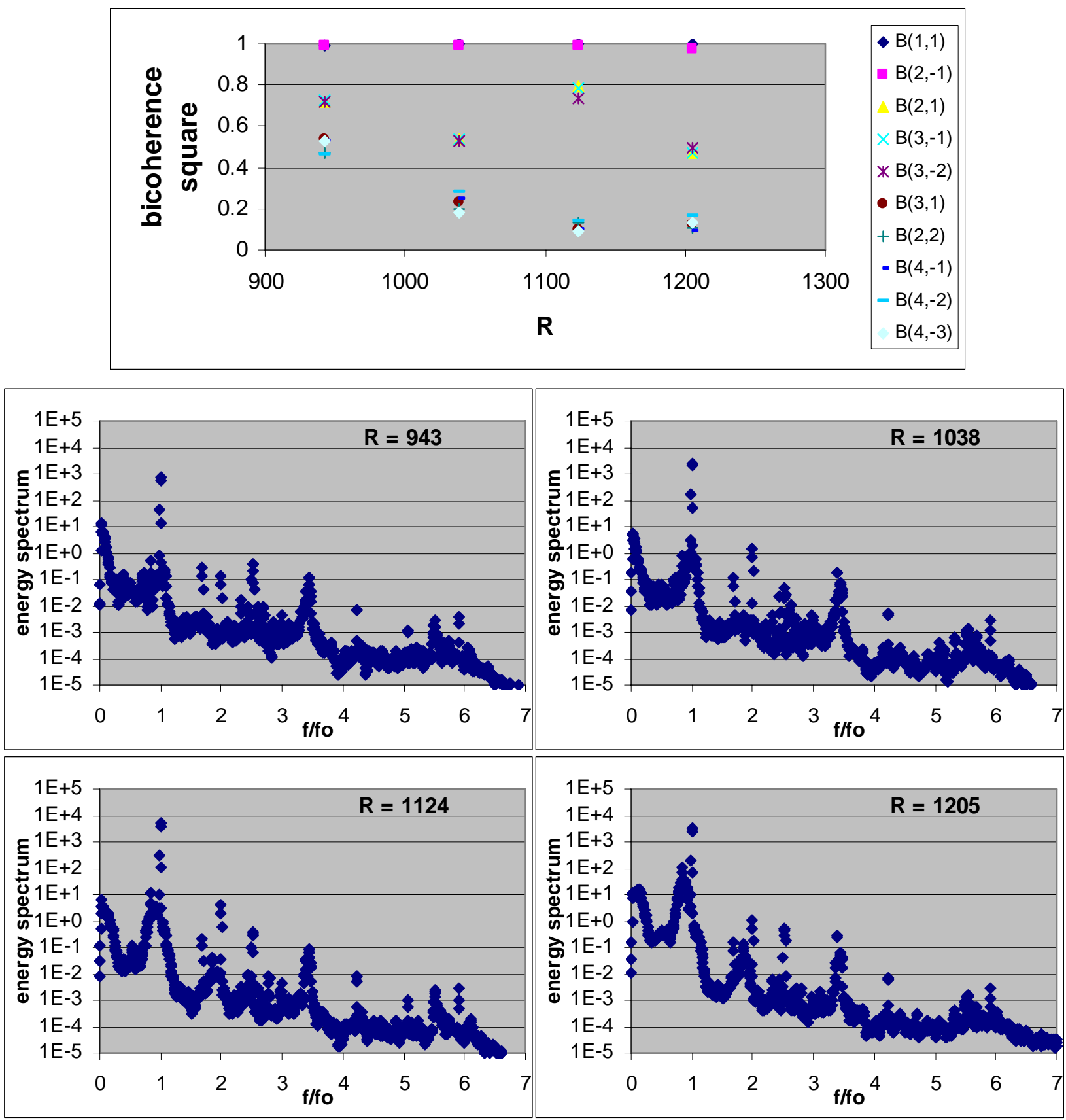

Figure 6. Bicoherence and energy spectrum as a function of $\mathbf{R}$ for the medium-excitation, medium-roughness case, $\psi=0^{\circ}$ (pattern B).

The results for the medium-excitation, medium-roughness case are shown in figure 6. Relative to the highexcitation, smooth-plate case (Fig. 3), the fundamental-mode amplitude is greater. The amplitudes of the modes at $\mathrm{f} / \mathrm{f}_{\mathrm{o}}=2$ and 3 are also greater, suggesting more effective energy transfer. In fact, for the $(1,1)$ and $(2,-1)$ triads, high bicoherence values persist farther, despite the lower excitation level. Roughness is evidently determinant here.

Relative to the medium-excitation, high-roughness case (Fig. 4), the medium-excitation, medium-roughness case shows lower fundamental and harmonic mode amplitudes, as well as lower bicoherence values for all but triads $(1,1)$ and $(2,-1)$. The mode at $\mathrm{f} / \mathrm{f}_{\mathrm{o}}=3$ loses amplitude, and the mode at $\mathrm{f} / \mathrm{f}_{\mathrm{o}}=4$ does not receive energy. Evidently there is no effective energy transfer to the higher harmonics, and therefore transition does not occur in the R region studied. 


\section{Case: Medium excitation, medium roughness, $\psi=15^{\circ}$ (pattern $M$ )}
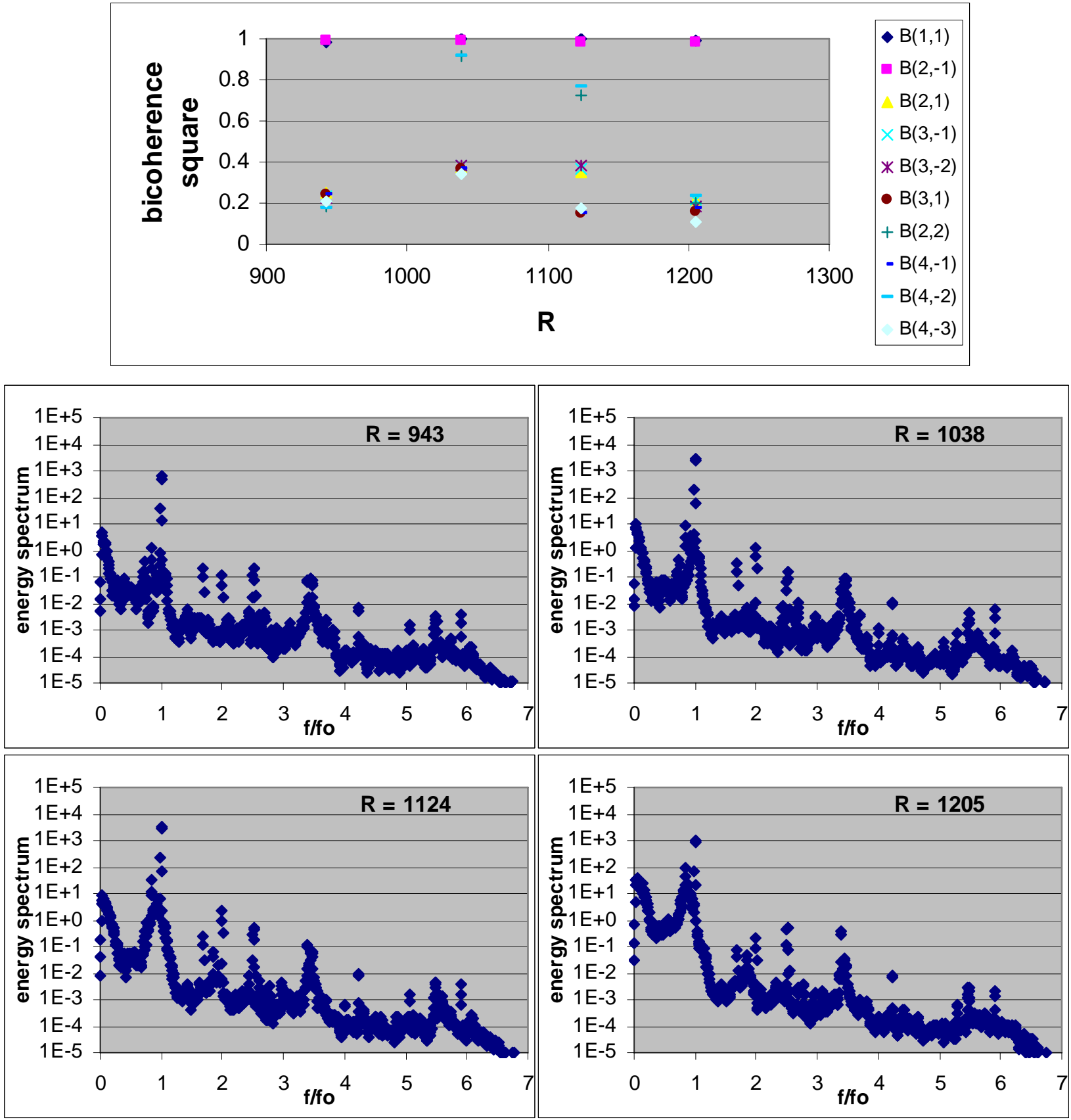

Figure 7. Bicoherence and energy spectrum as a function of $\mathbf{R}$ for the medium-excitation, medium-roughness case $\psi=15^{\circ}$ (pattern $\left.\mathrm{M}\right)$.

Figures 7, 8 and 9 show the results of the three-dimensional cases (obtained using with oblique surface roughness patterns) with $\psi=15^{\circ}, 30^{\circ}$ and $45^{\circ}$, respectively. The energy spectrum plots show that the fundamental mode amplitude decreases with increasing $\psi$. This behavior is consistent with the measurements by King and Breuer ${ }^{1}$ for oblique surface roughness. The case with $\psi=15^{\circ}$ (Fig. 7) is similar to the two-dimensional case of figure 6 . All but the $(1,1)$ and $(2,-1)$ triads have low bicoherence values. For the case with $\psi=30^{\circ}$ (Fig. 8) the bicoherence values of triads $(1,1)$ and $(2,-1)$ are lower at $\mathrm{R}=1124$ and 1205 . The effect is yet more pronounced for the case with $\psi=45^{\circ}$, (Fig. 9) with low bicoherence of triads $(1,1)$ and $(2,-1)$ at all but the first station. 


\section{Case: Medium excitation, medium roughness, $\psi=30^{\circ}$ (pattern $\mathrm{K}$ )}
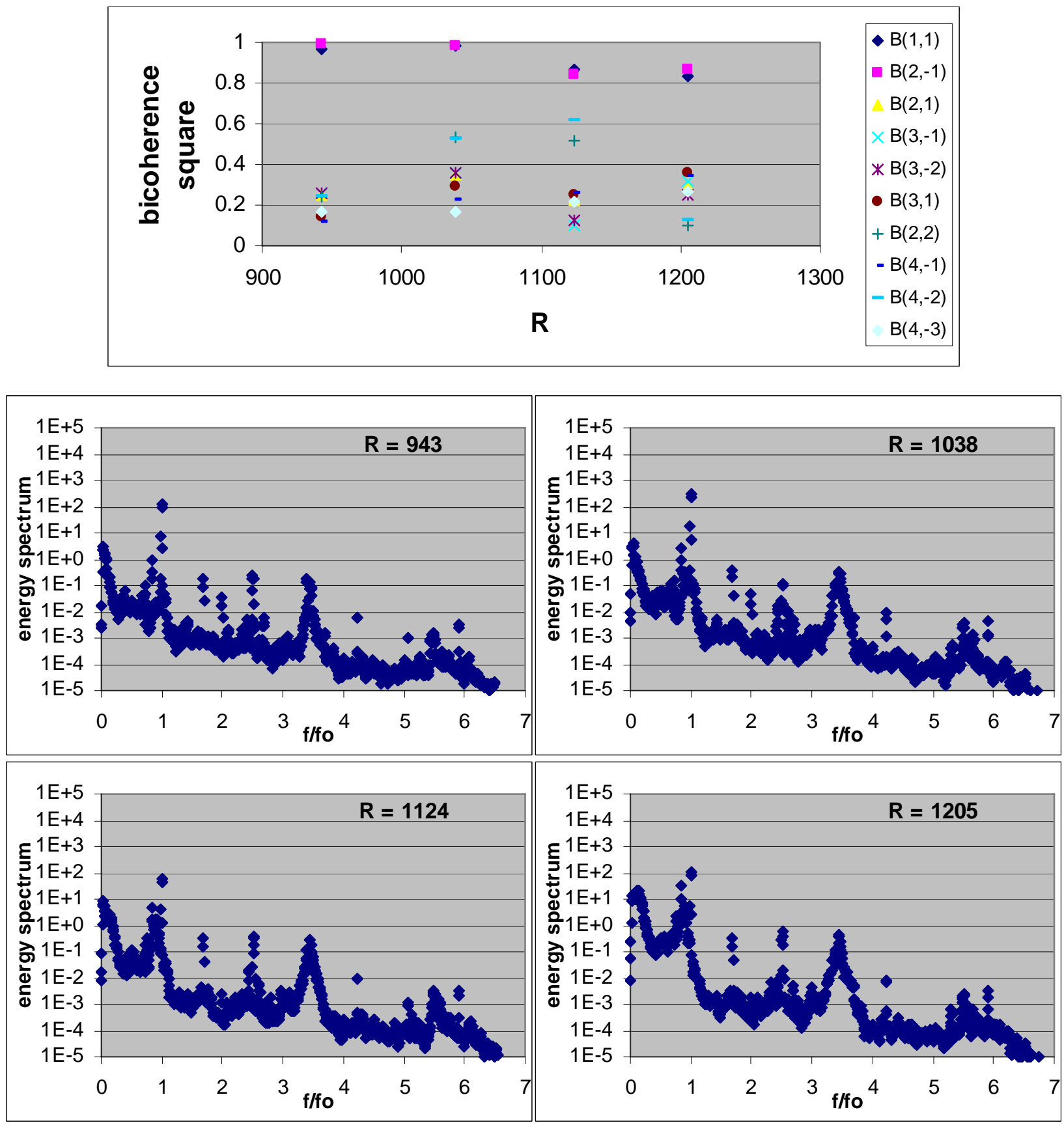

Figure 8. Bicoherence and energy spectrum as a function of $\mathbf{R}$ for the medium-excitation, medium-roughness case, $\psi=30^{\circ}$ (pattern $\mathrm{K}$ ).

In general, the bicoherence results reveal details of modal evolution not evident just from the energy spectrum. Two-dimensional roughness yields increased fundamental mode amplitude, and facilitates quadratic interactions that can lead to transition. To wit, the one case (Fig. 4) in which transition to turbulence appears incipient and the one case (Fig. 5) in which it occurs, are of high roughness. In both, the transition is preceded by strong interactions of all triads. In the medium excitation, medium roughness cases (Figs. 6 to 9) the energy spectrum and bicoherence results show that a larger orientation angle $\psi$ inhibits fundamental-mode growth and quadratic interaction of the selected triads. 


\section{Case: Medium excitation, medium roughness, $\psi=45^{\circ}$ (pattern $N$ )}
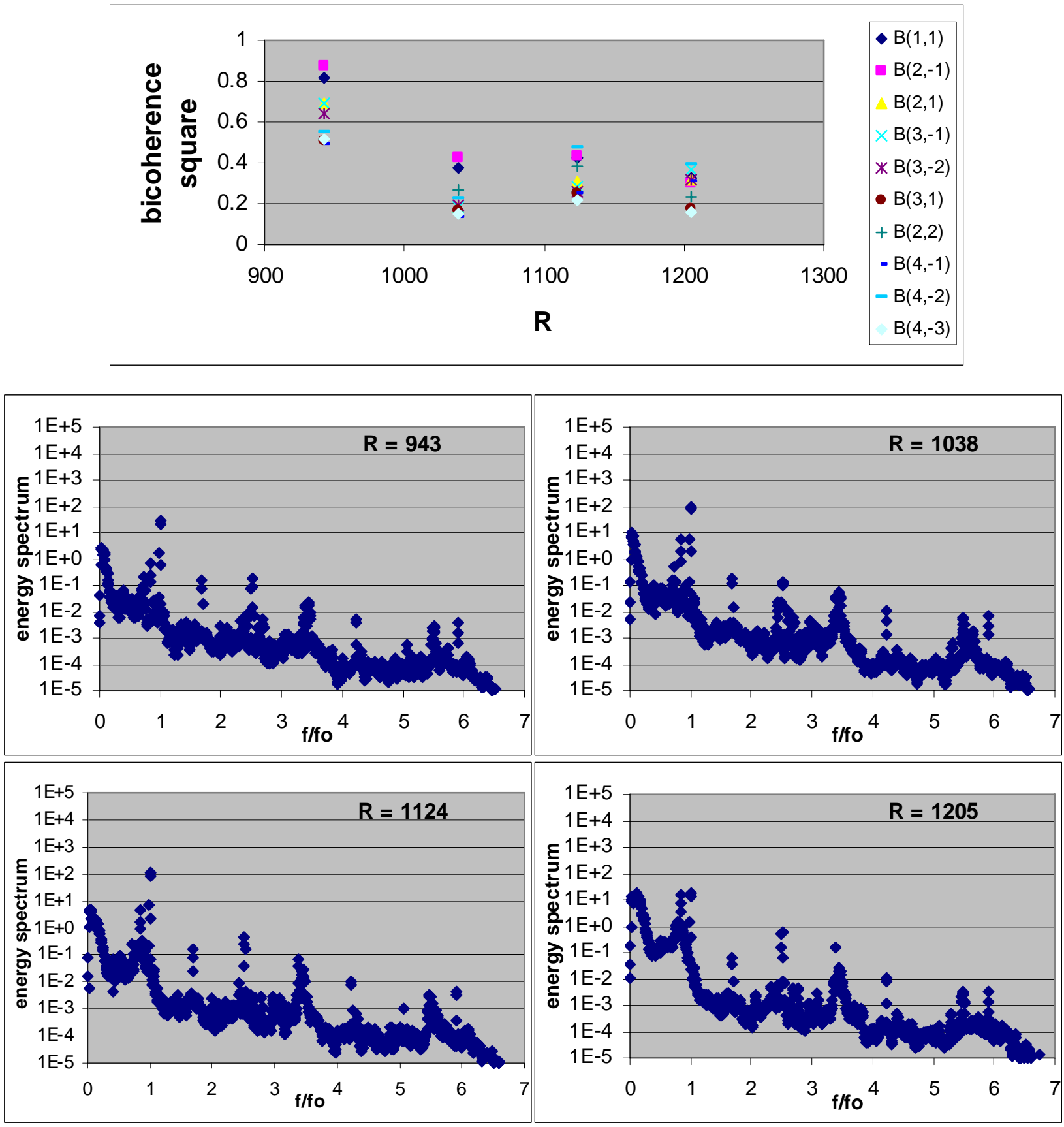

Figure 9. Bicoherence and energy spectrum as a function of $\mathbf{R}$ for the medium-excitation, medium-roughness case, $\psi=45^{\circ}$ (pattern $\left.N\right)$.

\section{Conclusion}

The results show that the roughness element thickness and orientation influence the development of quadratic interactions. Only part of the picture is evident from the evolution of energy spectrum values. In conjunction with these, bicoherence calculations can reveal a more complete picture of the evolution of interacting triads. 
In particular, the energy spectrum and bicoherence calculations show that: (1) in the two-dimensional case, higher surface roughness yields higher fundamental mode amplitudes and promotes quadratic interactions, and (2) that three-dimensionality imposed by surface roughness obliqueness inhibits quadratic interactions. The results confirm those of King and Breuer ${ }^{1}$. Also, they suggest that the presence of multiple, effective quadratic interactions are a precondition for transition in the $\mathrm{R}$ region studied.

\section{Acknowledgments}

J. B. Dávila thanks the Connecticut Space Grant and NASA EPSCoR for its financial support. Trinity College students Robert Philip Almquist and Robert Maloof worked on the production of energy spectrum and bicoherence plots.

\section{References}

${ }^{1}$ King, R. A. and Breuer, K. S., "Acoustic Receptivity and Evolution of Two-Dimensional and Oblique Disturbances in a Blasius Boundary Layer,” J. Fluid Mech., Vol. 432, 2001, pp. 69-90.

${ }^{2}$ Dávila, J. B., King, R. L. and Chokani, N., "Resonant Interactions of Instability Waves produced by Acoustic-Receptivity Mechanisms in a Blasius Boundary Layer," AIAA $43^{\text {rd }}$ Aerospace Sciences Meeting, Janurary, 2006.

${ }^{3}$ King, R. A., "Receptivity and Growth of Two- and Three-Dimensional Disturbances in a Blasius Boundary Layer," MIT, 2000.

${ }^{4}$ Kim, Y. C. and Powers, E. J., "Digital Bispectral Analysis and its Applications to Nonlinear Wave Interactions," IEEE Trans. Plasma Science, Vol. PS-7, 1979, pp. 120-131.

${ }^{5}$ Corke, T. C. and Mangano, R. A., "Resonant Growth of Three-Dimensional Modes in Transitioning Blasius Boundary Layers," J. Fluid Mech., Vol. 209, 1989, pp. 93-150.

${ }^{6}$ Miksad, R. W., Jones, F. L., Powers, E. J., Kim, Y. C. and Khadra, L, "Experiments on the Role of Amplitude and Phase Modulations during Transition to Turbulence," J. Fluid Mech., Vol. 123, 1982, pp. 1-29. 